\title{
Article
}

\section{A Bargaining Game with Proposers in the Hot Seat}

\section{Bram Driesen}

check for

updates

Citation: Driesen, B. A Bargaining Game with Proposers in the Hot Seat. Games 2021, 12, 87. https:/ /doi.org/ $10.3390 /$ g12040087

Academic Editors: Maria Gallego and Ulrich Berger

Received: 5 July 2021

Accepted: 4 November 2021

Published: 18 November 2021

Publisher's Note: MDPI stays neutral with regard to jurisdictional claims in published maps and institutional affiliations.

Copyright: (C) 2021 by the author. Licensee MDPI, Basel, Switzerland. This article is an open access article distributed under the terms and conditions of the Creative Commons Attribution (CC BY) license (https:/ / creativecommons.org/licenses/by/ $4.0 /)$.
Department Academic Affairs, Open University, Valkenburgerweg 177, 6419 AT Heerlen, The Netherlands; bram.driesen@ou.nl

\begin{abstract}
This note reconsiders the Rubinstein bargaining game under the assumption that a rejected offer is only costly to the proposer who made the rejected offer. It is shown that then, the classic result of Shaked that, in the multilateral version of this game, every division of the good can be sustained in SPE no longer holds. Specifically, there are many SPE, but players' (expected) payoffs in SPE are unique. The assumption further leads to a responder advantage.
\end{abstract}

Keywords: multilateral bargaining

JEL Classification: C78

\section{Introduction}

A feature of the classic alternating offers bargaining game of Rubinstein (1982) [1] is that at each stage, with some non-zero probability $1-\delta$, a rejected proposal leads to termination of the game, and all players receive a zero payoff. This set-up gives a distinct advantage to the proposing player: the risk of not receiving a payoff at all makes the responding players more amenable to accepting whatever is put on the table. This note examines a version of the game without this type of proposer advantage. In particular, we assume that with probability $1-\delta$, rejection terminates the game for the proposer, but not for the responders.

Such a modification to the original game can be motivated on empirical grounds. In particular, in the realm of political formation, responders do not face punishment for rejecting a proposal. After an election, it is typically the winner of that election-say, party A-who has the initiative to form a governing coalition. This party can be thought of as the proposer. It will make an offer to the parties it wishes to form a government with, and these parties can in turn accept or reject this proposal. Should party A's offer be found unreasonable and accordingly rejected, it is not uncommon for another party to take the initiative and to build a successful coalition from which party A is excluded. This is precisely what happened in the Belgian formation of 2019-2020. The Flemish nationalist party NVA received the highest share of the vote $(16 \%)$, and was therefore given the initiative to form a government. However, after their program was found to be unpalatable by the other parties, a government was eventually formed that excluded them [2].

Removing the cost of rejection has some non-trivial consequences. In the first place, Shaked's classic result that any partition of the pie can be sustained in SPE whenever there are three or more players (see [3] pp. 63-65) no longer holds. In our version of the game, there exist many SPE-even delay equilibria and equilibria that imply perpetual disagreement-but the expected payoff that players realize in these SPE is unique. Hence, given an immediate agreement, only one partition of the pie is feasible in SPE. A further consequence of our modeling choice is that there exists a proposer disadvantage. Players get to propose in a rotating order; the further a player is from being the proposer, the higher his/her expected payoff in the game. 


\section{The Game}

A set of players $N=\{1, \ldots, n\}$ is tasked with the division of a perfectly divisible unit good. At each period $t=0,1,2, \ldots$, in a rotating order $[1,2 \ldots, n, 1,2 \ldots]$, a player becomes the proposer; he/she gets to make a proposal or offer $z$ with $z_{i} \geq 0$ for all $i=1, \ldots, n$ and $z_{1}+\ldots+z_{n}=1$. The other players, the responders, simultaneously decide whether to accept or reject the proposer's offer. Whenever a time $t$ proposal $z$ is unanimously accepted by all responders, the proposed partition of the good is implemented, and the game ends; in such a case, each player $i$ 's resulting time $t$ utility is given by $z_{i}$. Whenever a player's time $t$ proposal is not unanimously accepted, it is rejected. In that case, the game continues to time $t+1$, where the next player in the above-described rotating order becomes the proposer. With probability $\delta(0<\delta<1)$, all players in $N$ remain in the game as the game moves to time $t+1$, and with probability $1-\delta$, the time $t$ proposer, say player $i$, is eliminated, receiving zero utility at time $t+1$. If $i$ is eliminated, the game continues at $t+1$ as described above with the player set $N \backslash i$, and player $i$ is henceforth skipped in the proposer order. An important distinction with the classic version of this game is that the expected utility of perpetual disagreement is well-defined, and not equal to zero.

\subsection{Preliminaries}

A time $t$ history, denoted $h^{t}$, specifies for all periods $s$ up until time $t$, the proposals that are made, and the coalition of players that survive period s. Specifically, $h^{t}=\left(\left(x^{0}, S\right), \ldots,\left(x^{t-1}, S^{\prime}\right)\right)$ where $\varnothing \subset S^{\prime} \subseteq S \subseteq N$. Furthermore, let $h^{0}$ be the empty history. The set of all time $t$ histories $h^{t}$ is denoted $H^{t}$.

A strategy $f_{i}$ for player $i$ is a set of functions $\left\{f_{i}^{t}\right\}_{t=0}^{\infty}$ that specifies player $i$ 's actions at each subgame, where he/she is still an active player. Let $Z^{S}$ be the set of proposals available to the players in $S \in 2^{N} \backslash \varnothing$-that is, $Z^{S}:=\left\{\left(z_{i}\right)_{i \in S} \mid z_{i} \geq 0\right.$ and $\left.\sum_{i \in S} z_{i}=1\right\}$ 一and let $Z:=\left\{Z^{S} \mid S \in 2^{N} \backslash \varnothing\right\}$. When $i$ is the proposer, $f_{i}^{t}: H^{t} \rightarrow Z$; that is, based on the history of play up until time $t$, player $i$ 's strategy prescribes which proposal he/she should make to the surviving coalition. When $i$ is a responder, $f_{i}^{t}: H^{t} \cup Z \rightarrow\{\mathrm{Y}, \mathrm{N}\}$; based on the play of the game up until time $t$, the current proposal on the table, and the time $t$ coalition of active players, player $i$ 's strategy specifies whether he/she should accept $(\mathrm{Y})$ or reject $(\mathrm{N})$. A tuple $f=\left(f_{1}, \ldots, f_{n}\right)$ specifying each player's strategy is called a strategy profile. Given a strategy profile $f, f_{-i}$ denotes the tuple of strategies played by players in $N \backslash i$.

The (expected) payoff a player $i \in N$ realizes when the strategy profile $f$ is played is denoted as $U_{i}(f)$. The payoff he/she realizes in the subgame that results from history $h^{t}$ is denoted $U_{i}\left(f \mid h^{t}\right)$.

A Nash equilibrium is a strategy profile $f$ such that for all $i \in N, U_{i}(f) \geq U_{1}\left(f_{i}^{\prime}, f_{-i}\right)$ for all strategies $f_{i}^{\prime}$. A subgame perfect equilibrium (SPE) is a strategy profile $f$ that is a Nash equilibrium in each subgame. More precisely, $U_{i}\left(f \mid h^{t}\right) \geq U_{i}\left(f_{i}^{\prime}\left|h^{t}, f_{-i}\right| h^{t}\right)$ for all strategies $f_{i}^{\prime}$ and histories $h^{t}$.

\subsection{The One-Shot Deviation Principle}

Since payoffs are not discounted, it is not immediate that the one-shot deviation principle applies. To see that it does, let $f$ and $f^{\prime}$ be two strategy profiles that coincide for periods 0 to $t-1$. Then, for all $i \in N$ and for all $h^{t} \in H^{t}$, the following holds:

$$
\left|U_{i}(f)-U_{i}\left(f^{\prime}\right)\right|=\delta^{t}\left|U_{i}\left(f \mid h^{t}\right)-U_{i}\left(f^{\prime} \mid h^{t}\right)\right| \leq \delta^{t} \cdot 1 .
$$

If under the strategy profile $f$, with some probability, a terminal node is reached prior to time $t$, then since $f^{\prime}$ coincides with $f$ prior to time $t$, this same terminal node is reached under $f^{\prime}$ with the same probability, and players realize the same physical payoffs. Hence, the expected payoff a player $i$ realizes from all terminal nodes prior to $t$ is the same under $f$ as it is under $f^{\prime}$, and in Equation (1), the two cancel each other out. Furthermore, the difference between the continuation values implied by two different strategy profiles is bounded from above by 1 at each subgame. From (1), it follows that for any $\varepsilon>0$, there is a 
$t$ such that for all strategy profiles $f, f^{\prime}$ coinciding for the first $t$ periods, $\left|U_{i}(f)-U_{i}\left(f^{\prime}\right)\right|<\varepsilon$. This condition, called continuity at infinity, is sufficient for the one-shot deviation principle to apply. That is, in verifying whether a strategy profile $f$ is SPE, we may restrict attention to one-period deviations from these strategies.

\section{The Two-Player Case}

In order to construct an SPE, assume that player 1 always proposes $x$ and 2 always proposes $y$, and that there is immediate agreement. If player 2 were to reject player 1 's proposal $x$, then in the next round, he/she obtains $y_{2}$ in case player 1 is not eliminated from the game (an event that occurs with probability $\delta$ ) and the entire pie if player 1 is eliminated from the game (an event that occurs with probability $(1-\delta)$ ). Similarly, if player 1 were to reject player 2's proposal $y$, then he/she realizes $x_{1}$ in case player 2 survives this rejection, and the entire pie otherwise.

$$
\begin{aligned}
& x_{2} \geq \delta y_{2}+(1-\delta) \cdot 1 \\
& y_{1} \geq \delta x_{1}+(1-\delta) \cdot 1 \\
& x_{1}+x_{2}=y_{1}+y_{2}=1
\end{aligned}
$$

Solving with equality yields the following:

$$
x=\left(\frac{\delta}{1+\delta}, \frac{1}{1+\delta}\right) \text { and } y=\left(\frac{1}{1+\delta}, \frac{\delta}{1+\delta}\right)
$$

This allows for the construction of an SPE. Consider the strategy profile $\hat{f}=\left(\hat{f}_{1}, \hat{f}_{2}\right)$, defined as follows:

$\hat{f}_{1}$ : As proposer, propose $x$; as responder, accept $v$ iff $v_{1} \geq y_{1}$.

$\hat{f}_{2}$ : As proposer, propose $y$; as responder, accept $v$ iff $v_{2} \geq x_{2}$.

Note that in this strategy profile, it is better to be a responder than a proposer.

Proposition 1. The strategy pair $\hat{f}$ is an SPE.

Proof. It is sufficient to show that $\hat{f}_{1}$ is optimal against $\hat{f}_{2}$ since the other case is analogous. Suppose, thus, that player 2 plays strategy $\hat{f}_{2}$. If player 1 is the proposer, following $\hat{f}_{1}$ yields $x_{1}$. If player 1 deviates by offering player 2 less than $x_{2}$, player 2 rejects and player 1 's payoff is $\delta y_{1}+(1-\delta) \cdot 0=x_{1}$. If player 1 deviates by offering player 2 more than $x_{2}$, player 2 accepts, and player 1's payoff is strictly below $x_{1}$. Hence, at even $t$, player 1 cannot profitably deviate from $\hat{f}_{1}$.

Consider an odd time $t$ at which player 1 is responding to a proposal $v$ by player 2 . By accepting, player 1 obtains $v_{1}$, while rejecting yields a payoff of $\delta x_{1}+(1-\delta) \cdot 1=y_{1}$. Hence, it is optimal for player 1 to accept player 2's proposal $v$ if $v_{1} \geq y_{1}$, and to reject it otherwise, as prescribed by $\hat{f}_{1}$.

It is clear that the value from perpetual disagreement in this model is not zero, like in the classical model. As it turns out, the players' expected payoffs from perpetual disagreement are given by the solution to system (2).

Lemma 1. Player $i$ 's $(i=1,2)$ time-t expected payoff from perpetual disagreement is $x_{i}$ if $t$ is even, and $y_{i}$ if $t$ is odd.

Proof. Note first that the values of perpetual disagreement in two identical subgames are the same. Thus, let $v=\left(v_{1}, v_{2}\right)$ be the players' values of perpetual disagreement at even $t$, and $w=\left(w_{1}, w_{2}\right)$ players' values of perpetual disagreement at odd $t$. Observe that for player 2 , the expected payoff from perpetual disagreement when he/she is the responder is 
equal to the expected payoff of his/her value of perpetual disagreement in the next round when he/she is the proposer. That is,

$$
v_{2}=\delta w_{2}+(1-\delta)
$$

Similarly, $w_{1}=\delta v_{1}+(1-\delta)$. Further note that the players' payoffs at every terminal node are either given by $(1,0)$ or $(0,1)$. Thus, $\left(v_{1}, v_{2}\right)=p(1,0)+(1-p)(0,1)$ for some probability $p$, which implies that $v_{1}+v_{2}=1$. Similarly, $w_{1}+w_{2}=1$. Thus, $v=x$ and $w=y$, as desired.

An immediate implication of Lemma 1 is that $\hat{f}$ is not the only SPE in this game. It even implies that perpetual disagreement can be supported in SPE. To see this, consider the profile $\tilde{f}=\left(\tilde{f}_{1}, \tilde{f}_{2}\right)$, defined as follows:

$\tilde{f}_{1}$ : As proposer, propose $x$; as responder, accept $v$ iff $v_{1}>y_{1}$.

$\tilde{f}_{2}$ : As proposer, propose $y$; as responder, accept $v$ iff $v_{2}>x_{2}$. an SPE.

Evidently, this strategy profile induces perpetual disagreement, but even so, it is

Proposition 2. The strategy pair $\left(\tilde{f}_{1}, \tilde{f}_{2}\right)$ is an SPE.

Proof. Again, it is sufficient to show that $\tilde{f}_{1}$ is optimal against $\tilde{f}_{2}$. Suppose that player 1 is the proposer. If he/she follows $\tilde{f}_{1}$, the result is perpetual disagreement, which by Lemma 1 yields $x_{1}$. If player 1 offers player 2 strictly less than $x_{2}$, player 2 rejects, leading to perpetual disagreement, and player 1's expected payoff continues to be $x_{1}$. If he/she offers player 2 strictly more than $x_{2}$, player 2 will accept, and player 1's payoff is strictly below $x_{1}$. Thus, as proposer, player 1 cannot profitably deviate from $\tilde{f}_{1}$.

Suppose on the other hand that player 2 is the proposer, and that he/she offers $v$. Rejecting $v$ again leads to perpetual disagreement. By Lemma 1 , player 1 's corresponding payoff is $y_{1}$. Thus, it is optimal for player 1 to accept $v$ if $v_{1}$ exceeds $y_{1}$, and to reject it otherwise, as prescribed by $\tilde{f}_{1}$.

Remark 1. It can be verified that $\left(\hat{f}_{1}, \tilde{f}_{2}\right)$ and $\left(\tilde{f}_{1}, \hat{f}_{2}\right)$ are SPE as well. We may even consider more intricate $\operatorname{SPE}\left(f_{1}, \tilde{f}_{2}\right)$, where player 1 starts out by playing $\tilde{f}_{1}$, and at some time $t$ switches to $\hat{f}_{1}$ so that the game concludes with an acceptance after several rounds of delay.

Remark 2. To see where the multiplicity of equilibria comes from, observe that when player 1 proposes $x$, player 2 is indifferent between accepting and rejecting. More precisely, accepting yields $x_{2}=1 /(1+\delta)$, while rejecting yields $\delta y_{2}+(1-\delta)=1 /(1+\delta)$. So what if, instead of accepting $x$, player 2 rejects? This can still be supported in SPE since player 1 has zero scope to make player 2 accept. Specifically, suppose that player 1 deviates by offering player 2 an $\varepsilon>0$ over and above $x_{2}$; player 2 will then certainly accept, but player 1's corresponding payoff is $\delta /(1+\delta)-\varepsilon$, which is strictly below the $\delta \cdot 1 /(1+\delta)+(1-\delta) \cdot 0=\delta /(1+\delta)$ he/she receives when player 2 rejects.

Fortunately, we can say something about the payoffs that players realize in SPE, which for our purposes is sufficient.

Proposition 3. In SPE, player i's $(i=1,2)$ expected time-t payoff is $x_{i}$ if $t$ is even, and $y_{i}$ if $t$ is odd.

Proof. For $i=1,2$, let $\bar{v}_{i}$ and $\underline{v}_{i}$, respectively, be the supremum and the infimum over all SPE of player $i$ 's continuation value in subgames where both players are in the game and $i$ is the proposer. Similarly, for $i=1,2$ let $\bar{w}_{i}$ and $\underline{w}_{i}$ respectively be the supremum and the infimum over all SPE of player $i$ 's continuation value in subgames where both players are in the game and $i$ is the responder. 
First note that at any time $t$, player 1 can commit to rejecting all of player 2's proposals when she is responding, and demanding $x_{1}$ when she is proposing. Thus, by Lemma 1 , she can unilaterally secure an (expected) payoff of $x_{1}$ when she is the proposer, and of $y_{1}$ when she is the responder. Therefore, $\underline{v}_{1} \geq x_{1}$ and $\underline{w}_{1} \geq y_{1}$. Similarly, $\underline{v}_{2} \geq y_{2}$ and $\underline{w}_{2} \geq x_{2}$.

In SPE, player 1 is never offered more than $\delta \bar{v}_{1}+(1-\delta)$ when 2 is the proposer. Whether player 2's proposal is accepted or rejected, player 1's payoff in SPE is, thus, at most $\delta \bar{v}_{1}+(1-\delta)$, that is, $\bar{w}_{1} \leq \delta \bar{v}_{1}+(1-\delta)$.

If player 1 is the proposer, player 2 's payoff is bounded from below by $\underline{w}_{2}$. Hence, player 1 can receive at most $1-\underline{w}_{2} \leq 1-x_{2}=x_{1}$ from an accepted offer. He/she can secure at most $\delta \bar{w}_{1}$ if his/her offer is rejected. Thus,

$$
\bar{v}_{1} \leq \max \left\{x_{1}, \delta \bar{w}_{1}\right\} \leq \max \left\{x_{1}, \delta^{2} \bar{v}_{1}+\delta(1-\delta)\right\}
$$

Then, either $\bar{v}_{1} \leq x_{1}$, or $\bar{v}_{1} \leq \delta^{2} \bar{v}_{1}+\delta(1-\delta)$, which also implies $\bar{v}_{1} \leq \delta /(1+\delta)=x_{1}$. Thus, in either case, $\bar{v}_{1}=\underline{v}_{1}=x_{1}$. Then $\bar{w}_{1} \leq \delta \bar{v}_{1}+(1-\delta)=\delta x_{1}+(1-\delta)=y_{1}$. Thus, $\bar{w}_{1}=\underline{w}_{1}=y_{1}$. By similar reasoning, $\bar{v}_{2}=\underline{v}_{2}=y_{2}$ and $\bar{w}_{2}=\underline{w}_{2}=x_{2}$.

The question is whether these results carry over to the multilateral case. Consider for a moment the classic model, where $\delta$ is interpreted as a discount factor so that a payoff $z$ realized at time $t$ yields utility $\delta^{t} z$. Shaked showed that if $1 / 2 \leq \delta<1$, then any partition of the good can be sustained in SPE in the three-player game. Let $e^{i}$ be the vector that gives one to player $i$, and zero to both $j \neq i$. Then the game is as follows.

In state $y=\left(y_{1}, y_{2}, y_{3}\right)$, each player $i$ proposes $y$, and accepts a proposal $x$ if and only if $x_{i} \geq \delta y_{i}$. If a player $i$ proposes $x$ with $x_{i}>y_{i}$, then the game moves immediately, prior to the responses of the other players, to the state $e^{j}$, where $j \neq i$ is the player with the lowest index for whom $x_{j}<1 / 2$. Note that there is at least one such player. To see that this is an SPE, consider first a player $i$ who responds to an offer $y$; rejecting yields $\delta y_{i}$, so accepting a proposal $x$ if and only if $x_{i} \geq \delta y_{i}$ is optimal. Consider next a player $i$ who proposes. The correct offer yields $y_{i}$. If $i$ proposes $x$ with $x_{i}>y_{i}$, the game switches to state $e^{j}$. Since $x_{j}<1 / 2 \leq \delta \cdot 1$, player $j$ rejects, and player $i$ obtains a zero payoff.

To see that in our version of the game, this strategy profile is not an SPE, assume that the game is in state $e^{1}$ and that player 2 is the proposer. Then, player 3 is correctly offered 0 , and receives zero payoff if he/she accepts. Rejecting, on the other hand, yields $\delta \cdot 0+(1-\delta) \frac{\delta}{(1+\delta)}$, which is strictly positive. Hence, for player 3 , in this subgame, the above-described strategy is not optimal, and as such, the profile is not an SPE.

\section{The $n$-Player Case $(n \geq 3)$}

In order to derive an SPE in the general game, note first that by symmetry, a player's (expected) payoff in the game is fully determined by his/her position, i.e., how many rounds it would take to become the proposer. The payoff is further completely independent from his / her identity. Let $w^{k}, k=0,1, \ldots, n-2$ be the (expected) payoff a player realizes in the game with $n-1$ players when he/she is $k$ rounds away from being the proposer. We assume that these are known quantities. Let $v^{0}, \ldots, v^{n-1}$ be the corresponding payoffs in the $n$-person game.

If a player is one period away from being the proposer, the (expected) payoff he/she realizes is $v^{1}$, which should be at least as good as going to the next round. With probability $\delta$, he/she becomes the proposer in the $n$-person game in this next period, in which case the (expected) payoff is $v^{0}$, while with probability $(1-\delta)$, he/she becomes the proposer in the $(n-1)$-person game that results from the proposer being eliminated from the game. In that case, the (expected) payoff is known and given by $w^{0}$. Hence, $v^{1} \geq \delta v^{0}+(1-\delta) w^{0}$. By similar reasoning, $v^{k} \geq \delta v^{k-1}+(1-\delta) w^{k-1}$ for $k>0$, and $v^{0} \geq \delta v^{n-1}$. Evaluating with equality, we obtain the following system: 


$$
\begin{aligned}
v^{1} & =\delta v^{0}+(1-\delta) w^{0} \\
\vdots & \\
v^{n-1} & =\delta v^{n-2}+(1-\delta) w^{n-2} \\
v^{0} & =\delta v^{n-1}
\end{aligned}
$$

We next show that this system has a unique solution, its components sum up to one, and if $\delta$ tends to one, the equal split is implemented. We further show that, even in the $n$-person game, there is proposer disadvantage. Specifically, the further a player is from being the proposer in the rotating order, the higher a payoff he/she realizes.

Proposition 4. The system (3) has a unique solution $v^{0}, \ldots, v^{n-1}$, for which $v^{0}+\ldots+v^{n-1}=1$, $v^{0}<\ldots<v^{n-1}$, and $\lim _{\delta \rightarrow 1} v^{k}=1 / n$ for all $k$.

Proof. Note that for the two-person case $v^{0}+v^{1}=1, v^{0}<v^{1}, v^{0}=\delta v^{1}$, and $\lim _{\delta \rightarrow 1}\left(v^{0}, v^{1}\right)$ $=(1 / 2,1 / 2)$. Assume that $w^{0}, \ldots, w^{n-2}$ satisfies the same properties:

A1. $w^{0}+\ldots+w^{n-2}=1$.

A2. $w^{0}<\ldots<w^{n-2}$.

A3. $w^{0}=\delta w^{n-2}$.

A4. For all $k=0, \ldots, n-2, \lim _{\delta \rightarrow 1} w^{k}=1 /(n-1)$.

Note that any solution to the system (3) trivially satisfies A3. Let $v^{0}, \ldots, v^{n-1}$ be a possible solution to system (3), and let $\sum_{k=0}^{n-1} v^{k}=q$. By A1, summing up the equations yields $q=\delta q+(1-\delta) \cdot 1$, and thus $q=1$. Hence, any solution to the system (3) satisfies A1. By A1 and the first $n-1$ equations of the system, we can uniquely determine $v^{0}$ :

$$
v^{0}=\frac{1-(1-\delta) \sum_{k=0}^{n-2} \sum_{t=0}^{n-k-2} \delta^{t} w^{k}}{\sum_{t=0}^{n-1} \delta^{t}}
$$

Hence, system (3) has a solution, and this solution is unique. Since $w^{0}, \ldots, w^{n-2}$ satisfies A4, the $w^{k}$ terms converge to finite values so that $\lim _{\delta \rightarrow 1} v^{0}=1 / n$. From this and, again, the fact that the $w^{k}$ terms converge to finite values, $\lim _{\delta \rightarrow 1} v^{k}=1 / n$ for all $k$.

To see that A2 is satisfied, assume, contrary to what we want, that $v^{n-1} \leq v^{n-2}$. Observe that

$$
v^{n-1}-v^{n-2}=\delta\left(v^{n-2}-v^{n-3}\right)+(1-\delta)\left(w^{n-2}-w^{n-3}\right) .
$$

Since $w^{0}, \ldots, w^{n-2}$ satisfies A2, $w^{n-2}-w^{n-3}>0$. Then by the initial assumption that $v^{n-1}-v^{n-2} \leq 0, v^{n-2}-v^{n-3} \leq 0$. By continuing this reasoning, $v^{n-3}-v^{n-4} \leq 0$, $v^{n-4}-v^{n-5} \leq 0, \ldots, v^{1}-v^{0} \leq 0$ so that

$$
v^{n-1}-v^{0}=v^{n-1}-v^{n-2}+v^{n-2}-v^{n-3}+\ldots+v^{1}-v^{0} \leq 0 .
$$

Since $v^{0}=\delta v^{n-1}$, we arrive at a contradiction. Thus, $v^{n-1}>v^{n-2}$. Then since $v^{n-1}=$ $\delta v^{n-2}+(1-\delta) w^{n-2}, v^{n-1}<w^{n-2}$, and thus by A3, $v^{0}<w^{0}$. Since $v^{1}=\delta v^{0}+(1-\delta) w^{0}$, this implies $v^{0}<v^{1}$. Since

$$
v^{2}-v^{1}=\delta\left(v^{1}-v^{0}\right)+(1-\delta)\left(w^{1}-w^{0}\right),
$$

it follows that $v^{1}<v^{2}$. Continuing this logic, $v^{0}<\ldots<v^{n-1}$, as desired.

Based on the solution to (3), construct the following strategy profile $\hat{f}$ : 
- A proposer claims $v^{0}$ for himself/herself, and offers $v^{k}$ to the player that is $k$ periods away from becoming the proposer.

- A responder $i, k$ periods away from becoming the proposer, accepts a proposal $x$ if and only if $x_{i} \geq v^{k}$.

Note that the proposals are feasible and efficient.

Proposition 5. The profile $\hat{f}$ is an SPE.

Proof. To see that the proposer rule is optimal, note that a rejected offer yields $\delta v^{n-1}=v^{0}$, and that the best a proposer can do from an accepted proposal is $v^{0}$.

Consider a responder who is $k$ periods from being the proposer. If all other players accept the proposer's offer, then rejecting yields $\delta v^{k-1}+(1-\delta) w^{k-1}=v^{k}$. Then, the responder rule is optimal.

Lemma 2. If at time $t$ a player is $k$ periods away from being the proposer, then their expected time $t$ payoff from perpetual disagreement is $v^{k}$.

Proof. Note first that a player's continuation values of perpetual disagreement in two identical subgames are the same. In addition, by symmetry of the players, a player's value of perpetual disagreement is fully determined by how many periods away they are from being the proposer. Thus, for $k=0, \ldots, n-1$, let $p^{k}$ be the value of perpetual disagreement of a player $k$ rounds from being the proposer. Note that the expected value of perpetual disagreement in the current round must be equal to the expected value of perpetual disagreement in the next round, i.e., $p^{0}=\delta p^{n-1}$ and $p^{k}=\delta p^{k-1}+(1-\delta) w^{k-1}$ if $k>0$. Then, $p^{k}=v^{k}$ for all $k$, as desired.

This result again implies that perpetual disagreement can be sustained in SPE. Let $\tilde{f}$ be the same as $\hat{f}$, except that the inequality in the accept/reject rule is strict. Such a profile leads to perpetual disagreement but is still an SPE.

Proposition 6. The profile $\tilde{f}$ is an SPE.

Proof. Trivial.

As before, this has the undesirable implication that there are a great many different SPEs. Not only is $\tilde{f}_{i}$ optimal against $\hat{f}_{-i}$, and vice versa, there are also more intricate SPEs where each player $i$ switches back and forth between $\hat{f}_{i}$ and $\tilde{f}_{i}$. However, using similar techniques as before, it is still the case that the payoffs that players realize in SPE are always the same.

Theorem 1. If a player is $k$ periods from being the proposer, their (expected) payoff in SPE is given by $v^{k}$.

Proof. Let $\bar{v}^{k}$ and $\underline{v}^{k}$ respectively be the supremum and the infimum over all SPE of a player's payoff when they are $k$ periods from becoming the proposer, and all players are in the game. Let $w^{k}$ be the (expected) payoff a player realizes in the subgame where he/she is $k$ periods from becoming the proposer, and one player has been eliminated from the game. By induction, we know that the equilibrium payoffs $w$ are unique in the game with $n-1$ players. Based on this assumption, we will argue that the equilibrium payoffs in the game with $n$ players are unique as well. In other words, $\bar{v}^{k}=\underline{v}^{k}$ for all $k=0, \ldots, n-1$.

Suppose that at time $t$ a player is $k$ periods from being the proposer, and all players are in the game. Since this player can commit to henceforth rejecting all proposals from his/her opponents and always demanding $v^{0}$ when they themselves are the proposer, a strategy that yields $v^{k}$ by Lemma 2 is $\underline{v}^{k} \geq v^{k}$. 
Since the payoffs of all responders are bounded from below by $\underline{v}^{k}$, an accepted proposal cannot give a proposer more than $1-\sum_{k=1}^{n-1} \underline{v}^{k}$. Furthermore, $\sum_{k=0}^{n-1} v^{k}=1$. Hence,

$$
\bar{v}^{0} \leq 1-\sum_{k=1}^{n-1} \underline{v}^{k} \leq 1-\sum_{k=1}^{n-1} v^{k}=v^{0}
$$

If the proposal is rejected, the proposer's expected payoff is bounded from above by the expected payoff of receiving $\bar{v}^{n-1}$ in the next round. That is, $\bar{v}^{0} \leq \delta \bar{v}^{n-1}$. Thus, the following holds:

$$
\bar{v}^{0} \leq \max \left\{v^{0}, \delta \bar{v}^{n-1}\right\} .
$$

Suppose a player is $k$ periods from being a proposer. The expected payoff from moving to the next round is $\delta \bar{v}^{k-1}+(1-\delta) w^{k-1}$, so he/she will never be offered more than that in the current round. Thus, whether the current proposal is accepted or rejected, this is an upperbound on the payoff that they realize in SPE in the current round. In other words,

$$
\bar{v}^{k} \leq \delta \bar{v}^{k-1}+(1-\delta) w^{k-1} \quad \text { for all } k=1, \ldots, n-1
$$

Since $v^{0}, \ldots, v^{n-1}$ is the unique solution to the system (3), it follows from (4) and (5) that $\bar{v}^{0} \leq v^{0}$. Then since $\bar{v}^{0} \geq \underline{v}^{0} \geq v^{0}, \bar{v}^{0}=\underline{v}^{0}=v^{0}$.

Let $k=1, \ldots, n-1$, and assume that $\bar{v}^{k-1}=v^{k-1}$. Then, by (5), $\bar{v}^{k} \leq \delta v^{k-1}+(1-$ $\delta) w^{k-1}=v^{k}$. Since $\bar{v}^{k} \geq \underline{v}^{k} \geq v^{k}, \bar{v}^{k}=\underline{v}^{k}=v^{k}$.

\section{Concluding Remarks}

In this paper, we departed from the classic alternating offers bargaining game of Rubinstein (1982) [1] by assuming that rejection is only costly to the proposer who made the rejected offer, and not to the responders who chose to reject. The implication of this modeling choice is that the (expected) payoffs in SPE are unique, even when the game features three or more players. This is in stark contrast with the classic version of the game, where every partition of the pie can be sustained in SPE (see [3] pp. 63-65). A further implication of our modeling choice is that we have a proposer disadvantage: the further a player is from being the proposer, the higher his/her expected payoff in the game.

These results depend crucially on the assumption that players have linear utility functions. When we assume that utility functions are concave, then the expected payoffs of perpetual disagreement are no longer efficient. Then, we are in more familiar waters-for two players, there is a unique SPE that entails immediate agreement; its corresponding payoffs converge to the Nash bargaining solution as $\delta$ tends to one. For three players, there may be other SPEs besides the stationary one. Specifically, we conjecture that there are SPEs that give the two responders their disagreement utility (i.e., the expected utility from perpetual disagreement) and the proposer the remainder of the pie.

Funding: This research received no external funding.

Institutional Review Board Statement: Not applicable.

Informed Consent Statement: Not applicable.

Data Availability Statement: Not applicable.

Conflicts of Interest: The authors declare no conflict of interest.

\section{References}

1. Rubinstein, A. Perfect equilibrium in a bargaining model. Econometrica 1982, 50, 97-109. [CrossRef]

2. Brzozowski, A. Belgium Breaks 16-Month Deadlock as Govenment Formed. Euractiv. 2020. Available online: https:// www.euractiv.com/section/politics/news/belgium-breaks-16-month-deadlock-as-new-government-formed/ (accessed on 20 September 2020).

3. Osborne, M.; Rubinstein, A. Bargaining and Markets; Academic Press: Cambridge, MA, USA, 1990. 